\title{
ON THE RELATIVE CLASS NUMBER OF SPECIAL CYCLOTOMIC FIELDS
}

\author{
TAKASHI AGOH
}

Dedicated to the memory of Tomihisa Oku

\begin{abstract}
Let $p$ be an odd prime, $\zeta_{p}$ be a primitive $p$ th root of unity and $h_{p}^{-}$be the relative class number of the $p$ th cyclotomic field $\mathbb{Q}\left(\zeta_{p}\right)$ over the rationals $\mathbb{Q}$ defined by $\zeta_{p}$. The main purpose of this paper is to discuss arithmetic properties of factors of $h_{p}^{-}$for an odd prime $p$ of the form $p=4 q+1$ with $q$ a prime.
\end{abstract}

\section{INTRODUCTION}

Let $p$ be an odd prime, $\zeta_{p}$ a primitive $p$ th root of unity, $K_{p}=\mathbb{Q}\left(\zeta_{p}\right)$ the cyclotomic field over the rationals $\mathbb{Q}$ defined $\zeta_{p}, h_{p}$ the class number of $K_{p}$ and $h_{p}^{+}$the real class number of $K_{p}$, i.e. the class number of the maximal real subfield $K_{p}^{+}=$ $\mathbb{Q}\left(\zeta_{p}+\zeta_{p}^{-1}\right) \subset K_{p}$. The relative class number $h_{p}^{-}=h_{p} / h_{p}^{+}$of $K_{p}$ as well as $h_{p}^{+}$has been the subject of considerable investigations in connection with the ideal class group of $K_{p}$ and many kinds of class number formulas have been devised from various viewpoints.

Out of numerous expressions of $h_{p}^{-}$, we first extract the following classical formula established by Kummer in 1851:

$$
h_{p}^{-}=\frac{(-1)^{(p-1) / 2}}{(2 p)^{(p-3) / 2}} \prod_{\substack{j=1 \\ j: \text { odd }}}^{p-1} f\left(\zeta_{p-1}^{j}\right),
$$

where $f(x)=\sum_{k=0}^{p-2} g_{k} x^{k}, g$ is a primitive root $(\bmod p)$ and $g_{k}$ is the least positive residue of $g^{k}$ modulo $p$. Next, we pick up the well-known

$$
h_{p}^{-}=2 p \prod_{\chi \in Z^{-}}\left(-\frac{1}{2} B_{1, \chi}\right),
$$

where $Z^{-}$is the set of all odd Dirichlet characters modulo $p$ and $B_{1, \chi}$ is the generalized Bernoulli number attached to $\chi$, i.e. $B_{1, \chi}=(1 / p) \sum_{a=1}^{p-1} a \chi(a)$.

Based on these formulas, we are able to deduce many important arithmetic properties of $h_{p}^{-}$(see, e.g., Ribenboim [14] and Washington [16]).

$M S C$ (2010): primary 11R04, 11R18, 11R29; secondary 11A07, 11R11.

Keywords: cyclotomic fields, class number formulas, relative class number.

The research was supported in part by the Grant-in-Aid for Scientific Research (C), Japan Society for the Promotion of Science. 
Concerning prime factors of $h_{p}^{-}$and $h_{p}^{+}$and their properties, very little is known. By elaborately analysing (1.1), Lehmer [7] obtained the following factorization of $h_{p}^{-}$into rational integers:

$$
h_{p}^{-}=\prod_{\substack{e d=p-1 \\ d: \text { odd }}} h_{p}(e)
$$

where the product is taken over all integers $e>0$ such that $e d=p-1$ with $d$ odd. The factor $h_{p}(e)$ is the so-called relative class number of order $e$. We can consult more details including an explicit formula of $h_{p}(e)$ in [7].

It is well-known by Kummer's result that $p\left|h_{p} \Leftrightarrow p\right| h_{p}^{-}, 2\left|h_{p} \Leftrightarrow 2\right| h_{p}^{-}$, $p\left|h_{p}^{+} \Rightarrow p\right| h_{p}^{-}$and $2\left|h_{p}^{+} \Rightarrow 2\right| h_{p}^{-}$. Metsänkylä [11] showed that if $p$ is a prime of the form $p=2 q+1$ with $q$ an odd prime, then $q \nmid h_{p}^{-}$. Concerning the parity of $h_{p}^{-}$, Estes [4] proved that if $p=2 q+1$ and 2 is inert in $K_{q}^{+}$, then $2 \nmid h_{p}^{-}$. We can find a new proof of this result in [12] based on the formula (1.2). See also the proof by Stevenhagen [15]. The parity of $h_{p}^{+}$was studied by Davis [3] and it was verified that if $p=2 q+1$ (both $p$ and $q$ are odd primes) and 2 is a primitive root of $q$, then $2 \nmid h_{p}^{+}$. On the one hand, Metsänkylä [13] discussed the case $p=4 q+1$ ( $q$ a prime) and proved a similar result to the Davis by making use of the 2 -adic class number formula.

In this paper, we focus our attention on the relative class number $h_{p}^{-}$of $K_{p}$ for an odd prime $p=4 q+1$ with $q$ a prime and discuss arithmetic properties of the factors $H_{1}$ and $H_{2}$ of $h_{p}^{-}$given in the following theorem.

Theorem 1.1. Let $p$ be an odd prime of the form $p=4 q+1$ with $q$ a prime. Then $h_{p}^{-}$is factored as $h_{p}^{-}=H_{1} \cdot H_{2}$, where $H_{1}$ and $H_{2}$ can be expressed by using integer pairs $(C, D)$ and $(S, T)$ as, respectively,

$$
H_{1}=\frac{C^{2}+D^{2}}{2} \text { and } H_{2}=\frac{S^{2}+(-1)^{(q-1) / 2} q T^{2}}{p} .
$$

Here the integers $C, D, S$ and $T$ are determined uniquely up to the sign.

We note that $H_{1}$ and $H_{2}$ in (1.4) are corresponding to $h_{p}(4)$ (with $d=q$ ) and $h_{p}(4 q)$ (with $d=1$ ), respectively, in Lehmer's factorization (1.3).

\section{Proof of Theorem 1.1}

Throughout this paper, we denote by $\zeta_{n}$ a primitive $n$th root of unity for $n \geq 1, \mathbb{Z}_{p}$ the ring of $p$-adic integers, $\mathbb{Q}_{p}$ the field of $p$-adic numbers, $\mathcal{O}_{K}$ the ring of integers in an algebraic number field $K$ over $\mathbb{Q}$ and $\mathcal{N}_{K / F}$ the norm in an extension $K / F$.

In this Section we first give the proof of Theorem 1.1 based on (1.2) and later we introduce methods how to concretely find the pairs $(C, D),(S, T)$ in (1.4) by making use of (1.1).

Proof. If we sort the odd characters $\chi$ according to their orders, then the numbers $\beta_{\chi}=-\frac{1}{2} B_{1, \chi}$ attached to $\chi \in Z^{-}$with $e=\operatorname{ord}(\chi)=(p-1) / d$ for a positive odd integer $d$ dividing $p-1$ form a Galois orbit in $K_{e}$. Therefore, letting $\alpha$ be an 
element in this orbit, we can write

$$
\mathcal{H}_{e}=\prod_{\operatorname{ord}(\chi)=e} \beta_{\chi}=\mathcal{N}_{K_{e} / \mathbb{Q}}(\alpha) \in \mathbb{Q}
$$

and hence (1.2) becomes

$$
h_{p}^{-}=2 p \prod_{\substack{e d=p-1 \\ d: \text { odd }}} \mathcal{H}_{e}
$$

This brings us Lehmer's factorization (1.3) if we perceive that the numbers $\beta_{\chi}=$ $-(1 / 2 p) \sum_{a=1}^{p-1} a \chi(a)$ are elements in $\mathcal{O}_{K_{p-1}}$ except for the following two cases.

(i) When $\operatorname{ord}(\chi)=e=2^{t}$ is the highest power of 2 dividing $p-1$, we know that $\beta_{\chi}$ is not 2-integral.

(ii) When $\operatorname{ord}(\chi)=e=p-1$ and $\chi$ is the inverse of the Teichmüller character $\omega$ of order $p-1$ after embedding $K_{p-1}$ in a fixed algebraic closure $\overline{\mathbb{Q}}_{p}$ of $\mathbb{Q}_{p}$, we see $\beta_{\chi} \in(1 / p) \mathbb{Z}_{p}$. Indeed, in this case we have $\beta_{\omega^{-1}}=\beta_{\omega^{p-2}} \equiv-(p-1) / 2 p\left(\bmod \mathbb{Z}_{p}\right)$, which corresponds to the von Staudt-Clausen theorem on Bernoulli numbers.

Therefore, taking $2 \mathcal{H}_{2^{t}}$ and $p \mathcal{H}_{p-1}$ together in (2.1), we have an actual factorization into rational integers as in (1.3). Now assume that $p$ is an odd prime of the form $p=4 q+1$ with $q$ a prime. Then we have $e=2^{t}=4$ and $e=p-1=4 q$, and hence $(2.1)$ can be written as $h_{p}^{-}=\left(2 \mathcal{H}_{4}\right)\left(p \mathcal{H}_{4 q}\right)$.

For the case $e=4$, we easily see that the number $H_{1}=2 \mathcal{H}_{4}$ can be written as, using a Gaussian integer $C+D i \in \mathcal{O}_{\mathbb{Q}(i)}=\mathbb{Z}[i]$,

$$
H_{1}=2 \prod_{\operatorname{ord}(\chi)=4} \beta_{\chi}=\frac{1}{2} \mathcal{N}_{\mathbb{Q}(i) / \mathbb{Q}}\left(2 B_{1, \chi}\right)=\frac{1}{2} \mathcal{N}_{\mathbb{Q}(i) / \mathbb{Q}}(C+D i)=\frac{C^{2}+D^{2}}{2} .
$$

For the case $e=4 q$, the number $H_{2}=p \mathcal{H}_{4 q}$ is equal to $p$ times the norm of an algebraic number which is integral in $K_{4 q}$ outside a single of the $\varphi(4 q)=2(q-1)$ primes over $p$ of valuation -1 , where $\varphi$ is Euler's totient function. Therefore, it is also $p$ times the norm of an algebraic integer which is integral outside a single of the 2 primes over $p$ of valuation -1 in any quadratic subfield of $K_{4 q}$, for which we may choose $F=\mathbb{Q}\left(\sqrt{(-1)^{\kappa} q}\right)$ with $\kappa=(q+1) / 2$. Writing $H_{2}$ as $1 / p$ times the norm of $p$ times that quadratic number which is integral in $F$, we arrive at the expression of $H_{2}$ by means of a pair $(S, T) \in \mathbb{Z}^{2}$ as follows:

$$
H_{2}=\frac{1}{p} \mathcal{N}_{F / \mathbb{Q}}\left(S+\sqrt{(-1)^{\kappa} q} T\right)=\frac{S^{2}+(-1)^{(q-1) / 2} q T^{2}}{p} .
$$

This completes the proof of Theorem 1.1.

Above proof based on (1.2) tells that there exist pairs $(C, D)$ and $(S, T)$ of integers as stated in (1.4), however it does not show the uniqueness of these pairs. Further, an algorithm how to concretely deduce them does not come in sight. These issues will be resolved when we apply Kummer's formula (1.1). We do not give details because it is rather lengthy and troublesome, but we think that it is better to introduce below only the process how to find explicitly these pairs by making use of (1.1). 
As defined in Section 1, let $f(x)=\sum_{k=0}^{p-2} g_{k} x^{k}$, where $g^{k} \equiv g_{k}(\bmod p), 1 \leq$ $g_{k} \leq p-1$, for a primitive root $g(\bmod p)$. Then we have $f(x)=\sum_{k=1}^{4 q} k x^{\text {ind }(k)}$ if $p=4 q+1$. Since $\{a \in \mathbb{Z} \mid 1 \leq a<4 q,(a, 4 q)=1\}=\{2 k+1 \mid 0 \leq k<2 q\} \backslash\{q, 3 q\}$,

$$
\mathcal{N}_{K_{4 q} / \mathbb{Q}}\left(f\left(\zeta_{4 q}\right)\right)=\prod_{\substack{a=1 \\(a, 4 q)=1}}^{4 q-1} f\left(\zeta_{4 q}^{a}\right) .
$$

As easily seen, $\zeta_{4 q}=i \zeta_{q}, \zeta_{4 q}^{q}=i^{q}=(-1)^{(q-1) / 2} i, \zeta_{4 q}^{3 q}=-i^{q}=(-1)^{(q+1) / 2} i$ and $\left\{i^{q}, i^{3 q}\right\}=\{i,-i\}$ for $i=\sqrt{-1}$. Since $K_{4}=\mathbb{Q}(i)$, we obtain from (1.1)

$$
\begin{aligned}
h_{p}^{-} & =\frac{1}{(2 p)^{2 q-1}} \prod_{k=0}^{2 q-1} f\left(\left(i \zeta_{q}\right)^{2 k+1}\right) \\
& =\frac{1}{(2 p)^{2 q-1}}\left\{\mathcal{N}_{K_{4} / \mathbb{Q}}(f(i)) \cdot \mathcal{N}_{K_{4 q} / \mathbb{Q}}\left(f\left(i \zeta_{q}\right)\right)\right\} .
\end{aligned}
$$

Here we write $h_{p}^{-}$as $h_{p}^{-}=H_{1} \cdot H_{2}$, where

$$
H_{1}=\frac{1}{2 p^{2}} \mathcal{N}_{K_{4} / \mathbb{Q}}(f(i)) \text { and } H_{2}=\frac{1}{2^{2(q-1)} p^{2 q-3}} \mathcal{N}_{K_{4 q} / \mathbb{Q}}\left(f\left(i \zeta_{q}\right)\right) .
$$

For an appropriate function $\vartheta$ and $a=0,1,2,3$, let write for simplification

$$
\sum_{k}^{(a)} \vartheta(k)=\sum_{\substack{k=1 \\ \operatorname{ind}(k) \equiv a(\bmod 4)}}^{p-1} \vartheta(k) .
$$

First we put $U_{a}=\sum_{k}^{(a)} k$. Since $\operatorname{ind}(k) \equiv a(\bmod 4)$ deduces $\operatorname{ind}(p-k)=$ $\operatorname{ind}(-k) \equiv a+2(\bmod 4)$, we have $U_{a+2}=\sum_{k}^{(a+2)} k=\sum_{k}{ }^{(a)}(p-k)=q p-U_{a}$ for $a=0,1$. Also since

$$
\begin{aligned}
f(i) & =\sum_{k=0}^{p-2} g_{k} i^{k}=\sum_{k=1}^{4 q} k i^{\operatorname{ind}(k)}=\sum_{a=0}^{3} \sum_{k}^{(a)} k i^{\operatorname{ind}(k)} \\
& =\left(U_{0}-U_{2}\right)+i\left(U_{1}-U_{3}\right)=\left(2 U_{0}-p q\right)+i\left(2 U_{1}-p q\right),
\end{aligned}
$$

we get from $(2.2)$

$$
\begin{aligned}
H_{1} & =\frac{1}{2 p^{2}} f(i) f(-i)=\frac{1}{2 p^{2}}\left\{\left(2 U_{0}-p q\right)^{2}+\left(2 U_{1}-p q\right)^{2}\right\} \\
& =\frac{1}{2}\left\{\left(2 U_{0} / p-q\right)^{2}+\left(2 U_{1} / p-q\right)^{2}\right\} .
\end{aligned}
$$

Noting that $U_{a} \equiv \sum_{k}^{(a)} g^{\operatorname{ind}(k)} \equiv \sum_{k=0}^{q-1} g^{a+4 k} \equiv g^{a}\left(g^{p-1}-1\right) /\left(g^{4}-1\right) \equiv 0(\bmod p)$ by Fermat's little theorem, if we set $C=\left|2 U_{0} / p-q\right|$ and $D=\left|2 U_{1} / p-q\right|$, then (2.3) leads to $H_{1}=\left(C^{2}+D^{2}\right) / 2$ with $(C, D) \in \mathbb{Z}^{2}$ as indicated in (1.4).

Next put $V_{a}=\sum_{k}^{(a)} k \zeta_{q}^{\text {ind }(k)}$ for $a=0,1,2,3$. Then it follows that for $a=0,1$

$$
\begin{aligned}
V_{a+2} & =\sum_{k}^{(a+2)}(p-k) \zeta_{q}^{\operatorname{ind}(p-k)}=\sum_{k}^{(a)}(p-k) \zeta_{q}^{\operatorname{ind}(-k)} \\
& =\sum_{k}^{(a)}(p-k) \zeta_{q}^{\text {ind }(k)}=p \sum_{k}^{(a)} \zeta_{q}^{\operatorname{ind}(k)}-\sum_{k}^{(a)} k \zeta_{q}^{\text {ind }(k)}=-V_{a} .
\end{aligned}
$$


Thus we have

$$
\begin{aligned}
f\left(i \zeta_{q}\right) & =\sum_{k=1}^{4 q} k\left(i \zeta_{q}\right)^{\operatorname{ind}(k)}=\sum_{a=0}^{3} \sum_{k}^{(a)} k\left(i \zeta_{q}\right)^{\operatorname{ind}(k)} \\
& =\left(V_{0}-V_{2}\right)+i\left(V_{1}-V_{3}\right)=2\left(V_{0}+i V_{1}\right) .
\end{aligned}
$$

Noting the fact $K_{4 q}=K_{q}(i)$, if we calculate the norm of $f\left(i \zeta_{q}\right)$ in $K_{4 q} / \mathbb{Q}$, then

$$
\begin{aligned}
\mathcal{N}_{K_{4 q} / \mathbb{Q}}\left(f\left(i \zeta_{q}\right)\right) & =2^{2(q-1)} \mathcal{N}_{K_{4 q} / \mathbb{Q}}\left(V_{0}+i V_{1}\right) \\
& =2^{2(q-1)} \mathcal{N}_{K_{q} / \mathbb{Q}}\left(\mathcal{N}_{K_{q}(i) / K_{q}}\left(V_{0}+i V_{1}\right)\right) \\
& =2^{2(q-1)} \mathcal{N}_{K_{q} / \mathbb{Q}}\left(V_{0}^{2}+V_{1}^{2}\right) .
\end{aligned}
$$

Putting afresh $\alpha_{1}=V_{0}$ and $\beta_{1}=V_{1}$, we define $\alpha_{j+1}=\Gamma^{j}\left(\alpha_{1}\right)$ and $\beta_{j+1}=\Gamma^{j}\left(\beta_{1}\right)$ $(j=0,1, \ldots, q-2)$, where $\Gamma$ is a generator of $\operatorname{Gal}\left(K_{q} / \mathbb{Q}\right)$. Consider the recurrence sequences $\left\{X_{n}\right\}_{n \geq 1}$ and $\left\{Y_{n}\right\}_{n \geq 1}$ defined by $X_{1}=\alpha_{1}, Y_{1}=\beta_{1}$ and for $k \geq 1$

$$
\left\{\begin{array}{c}
X_{k+1}=\alpha_{k+1} X_{k}+\beta_{k+1} Y_{k}, \\
Y_{k+1}=\beta_{k+1} X_{k}-\alpha_{k+1} Y_{k} .
\end{array}\right.
$$

Then we see $X_{q-1}=\Gamma^{j}\left(X_{q-1}\right)$ and $Y_{q-1}=(-1)^{j} \Gamma^{j}\left(Y_{q-1}\right)$ for any $j \geq 0$, which show $X_{q-1} \in \mathbb{Z}$ and $Y_{q-1} \in \mathcal{O}_{F}$, where $F=\mathbb{Q}\left(\sqrt{(-1)^{\rho} q}\right) \subset K_{q}$ and $\rho=(q-1) / 2$. When we represent $Y_{q-1}$ using the integral basis $\left\{1, \frac{1}{2}\left(1+\sqrt{(-1)^{\rho} q}\right)\right\}$ of $\mathcal{O}_{F}$, there exist uniquely $u_{q}, v_{q} \in \mathbb{Z}$ such that

$$
Y_{q-1}=u_{q}+v_{q} \frac{1+\sqrt{(-1)^{\rho} q}}{2}
$$

We do not mention details, but it can be shown that $Y_{q-1}=-u_{q} \sqrt{(-1)^{\rho} q}$ based on the fact $v_{q}=-2 u_{q}$. Putting anew $X=X_{q-1}$ and $Y=u_{q}=-Y_{q-1} / \sqrt{(-1)^{\rho} q}$, we see that $X, Y \in \mathbb{Z}$ and both are divisible by $p^{q-2}$. Consequently, letting $S=$ $\left|X / p^{q-2}\right|$ and $T=\left|Y / p^{q-2}\right|$, we realize the following expression of $H_{2}$ as desired:

$$
\begin{aligned}
H_{2} & =\frac{1}{2^{2(q-1)} p^{2 q-3}} \mathcal{N}_{K_{4 q} / \mathbb{Q}}\left(f\left(i \zeta_{q}\right)\right)=\frac{1}{p^{2 q-3}} \mathcal{N}_{K_{q} / \mathbb{Q}}\left(V_{0}^{2}+V_{1}^{2}\right) \\
& =\frac{1}{p^{2 q-3}} \prod_{j=0}^{q-2} \Gamma^{j}\left(\alpha_{1}^{2}+\beta_{1}^{2}\right)=\frac{1}{p^{2 q-3}} \prod_{k=1}^{q-1}\left(\alpha_{k}^{2}+\beta_{k}^{2}\right) \\
& =\frac{X_{q-1}^{2}+Y_{q-1}^{2}}{p^{2 q-3}}=\frac{X^{2}+(-1)^{(q-1) / 2} q Y^{2}}{p^{2 q-3}} \\
& =\frac{S^{2}+(-1)^{(q-1) / 2} q T^{2}}{p} .
\end{aligned}
$$

\section{Arithmetic properties of $H_{1}$ And $H_{2}$}

In this Section, we discuss arithmetic properties of the factors $H_{1}$ and $H_{2}$ of $h_{p}^{-}$ stated in Theorem 1.1.

First, we shall prove the following

Proposition 3.1. Let $p=4 q+1$ be an odd prime with $q$ a prime, $H_{1}, H_{2}$ be as in Theorem 1.1 and $(\dot{q})$ be the Legendre symbol. Then we have 
(i) $H_{1} \equiv 1(\bmod 4)$.

(ii) If $\mathrm{H}_{2}$ is odd, then $\mathrm{H}_{2} \equiv 1(\bmod 4)$.

(iii) If $q \nmid H_{2}$, then $\left(\frac{H_{2}}{q}\right)=1$.

(iv) If $l$ is an odd prime with $l \neq q$ and $l \| H_{2}$, then $\left(\frac{l}{q}\right)=(-1)^{(l-1) / 2}$.

Proof. (i) Recall the expression $H_{1}=\left(C^{2}+D^{2}\right) / 2$ from Section 2. Since both $C=\left|2 U_{0} / p-q\right|$ and $D=\left|2 U_{1} / p-q\right|$ are odd, we see $C^{2}+D^{2} \equiv 2(\bmod 8)$ and hence $H_{1} \equiv 1(\bmod 4)$.

(ii) From the assumption $2 \nmid H_{2}$ and the expression of $H_{2}$ in (2.6), $S$ and $T$ must have different parities. If $S$ is odd and $T$ is even, then $H_{2} \equiv S^{2} \equiv 1(\bmod 4)$. On the one hand, if $S$ is even and $T$ is odd, then $H_{2} \equiv(-1)^{(q-1) / 2} q T^{2} \equiv 1(\bmod 4)$, because it always holds that $(-1)^{(q-1) / 2} q \equiv 1(\bmod 4)$ for an odd prime $q$.

(iii) Since $p \equiv 1(\bmod q)$ and $p H_{2}=S^{2}+(-1)^{(q-1) / 2} q T^{2}$, we have $H_{2} \equiv S^{2}$ $(\bmod q)$ and hence $\left(\frac{H_{2}}{q}\right)=1$.

(iv) If $l=p$, then $\left(\frac{l}{q}\right)=\left(\frac{4 q+1}{q}\right)=1$, which proves the assertion. On the other hand, if $l \neq p, q$, then, by the reciprocity and the first complementary laws for the Legendre symbol, it follows that, letting $\kappa=(q+1) / 2$,

$$
\begin{aligned}
l \text { is inert in } \mathbb{Q}\left(\sqrt{(-1)^{\kappa} q}\right) & \Longleftrightarrow\left(\frac{(-1)^{\kappa} q}{l}\right)=-1 \\
& \Longleftrightarrow\left(\frac{l}{q}\right)=(-1)^{(l+1) / 2} .
\end{aligned}
$$

If $\left(\frac{l}{q}\right) \neq(-1)^{(l-1) / 2}$, i.e. $\left(\frac{l}{q}\right)=(-1)^{(l+1) / 2}$, then we know from (3.1) that $l$ is inert in $\mathbb{Q}\left(\sqrt{(-1)^{\kappa} q}\right)$. However $H_{2}$ can be written as

$$
H_{2}=\frac{1}{p}\left(S+\sqrt{(-1)^{\kappa} q} T\right)\left(S-\sqrt{(-1)^{\kappa} q} T\right),
$$

which implies that if $l \mid H_{2}$, then $l^{2} \mid H_{2}$. This is contrary to $l \| H_{2}$.

Combining (i) and (ii) in Proposition 3.1, we know that if $h_{p}^{-}$is odd, then $h_{p}^{-} \equiv 1(\bmod 4)$.

Proposition 3.2. Let $p=4 q+1$ be an odd prime, where $q$ is also a prime with $q \equiv 3(\bmod 4)$. Then we have (i) $H_{1} \not \equiv 0(\bmod q)$ and $(\mathrm{ii}) H_{2} \not \equiv 0(\bmod q)$, and hence $h_{p}^{-} \not \equiv 0(\bmod q)$.

Proof. (i) We shall first show $H_{1} \not \equiv 0(\bmod q)$ if $q \equiv 3(\bmod 4)$. Let $U_{a}$ be as in Section 2. Then, since $U_{a}>0, U_{a} \equiv 0(\bmod p)$ and $U_{a}+U_{a+2}=p q$, we know $\left(U_{a}, q\right)=1$ for each $i=0,1,2,3$. In fact, if $q \mid U_{a}$, then $U_{a} / p q+U_{a+2} / p q=1$, which is impossible because both $U_{a} / p q$ and $U_{a+2} / p q$ are positive integers. Recall now the expression of $H_{1}$ introduced in Section 2 as a consequence of (1.1):

$$
H_{1}=\frac{C^{2}+D^{2}}{2}
$$


where $C=\left|2 U_{0} / p-q\right|$ and $D=\left|2 U_{1} / p-q\right|$. Since $\left(U_{0}, q\right)=\left(U_{1}, q\right)=1$, one knows $(C, q)=(D, q)=1$. This implies that if $H_{1} \equiv 0(\bmod q)$, then $q \equiv 1(\bmod 4)$, which is contrary to the assumption.

(ii) Next, we shall show $H_{2} \not \equiv 0(\bmod q)$ if $q \equiv 3(\bmod 4)$. Let $\alpha_{1}=V_{0}$ and $\beta_{1}=V_{1}$ as defined in Section 2 and put $\mathfrak{q}=\left(1-\zeta_{q}\right)$ the prime ideal of $\mathcal{O}_{K_{q}}$ dividing $q$. Then we have $\alpha_{1} \equiv U_{0}(\bmod \mathfrak{q})$ and $\beta_{1} \equiv U_{1}(\bmod \mathfrak{q})$, because $U_{a}-V_{a}=\sum_{k}^{(a)} k\left(1-\zeta_{q}^{\text {ind }(k)}\right) \equiv 0(\bmod \mathfrak{q})$ for $a=0,1$. Hence, letting $\alpha_{j+1}=$ $\Gamma^{j}\left(\alpha_{1}\right)$ and $\beta_{j+1}=\Gamma^{j}\left(\beta_{1}\right)$ for $\Gamma \in \operatorname{Gal}\left(K_{q} / \mathbb{Q}\right)$, it follows that for $j=0,1, \ldots, q-2$

$$
\alpha_{j+1} \equiv U_{0} \quad(\bmod \mathfrak{q}), \quad \beta_{j+1} \equiv U_{1}(\bmod \mathfrak{q}) .
$$

Here reconsider the sequences $\left\{X_{n}\right\}_{n \geq 1}$ and $\left\{Y_{n}\right\}_{n \geq 1}$ defined in (2.5). From (3.2) we deduce for any $k \geq 1$

$$
\left\{\begin{array} { l } 
{ X _ { 2 k - 1 } \equiv ( U _ { 0 } ^ { 2 } + U _ { 1 } ^ { 2 } ) ^ { k - 1 } U _ { 0 } ( \operatorname { m o d } \mathfrak { q } ) , } \\
{ Y _ { 2 k - 1 } \equiv ( U _ { 0 } ^ { 2 } + U _ { 1 } ^ { 2 } ) ^ { k - 1 } U _ { 1 } ( \operatorname { m o d } \mathfrak { q } ) , }
\end{array} \quad \left\{\begin{array}{l}
X_{2 k} \equiv\left(U_{0}^{2}+U_{1}^{2}\right)^{k} \quad(\bmod \mathfrak{q}) \\
Y_{2 k} \equiv 0(\bmod \mathfrak{q})
\end{array}\right.\right.
$$

Taking account of the facts $X_{q-1}, Y_{q-1}^{2} \in \mathbb{Z}$ and $(q)=\mathfrak{q}^{q-1}$, we get from (3.3)

$$
X_{q-1} \equiv\left(U_{0}^{2}+U_{1}^{2}\right)^{\rho}(\bmod q), \quad Y_{q-1}^{2} \equiv 0(\bmod q),
$$

where $\rho=(q-1) / 2$. As mentioned in Section $2, H_{2}$ can be expressed as

$$
H_{2}=\frac{S^{2}+(-1)^{\rho} q T^{2}}{p},
$$

where $S, T$ are rational integers given by

$$
S=\left|\frac{X_{q-1}}{p^{q-2}}\right| \text { and } T=\left|\frac{-Y_{q-1}}{p^{q-2} \sqrt{(-1)^{\rho} q}}\right| .
$$

If we assume $H_{2} \equiv 0(\bmod q)$ for $q \equiv 3(\bmod 4)$, then $S \equiv 0(\bmod q)$ since $p \equiv 1$ $(\bmod q)$. Therefore, from $(3.4)$ we get $X_{q-1} \equiv\left(U_{0}^{2}+U_{1}^{2}\right)^{\rho} \equiv 0(\bmod q)$ which implies $U_{0}^{2}+U_{1}^{2} \equiv 0(\bmod q)$. Also since $\left(U_{0}, q\right)=\left(U_{1}, q\right)=1, q$ must satisfy $q \equiv 1(\bmod 4)$, which is however contrary to the assumption.

By (i) and (ii), we conclude that $h_{p}^{-}=H_{1} \cdot H_{2} \not \equiv 0(\bmod q)$ if $q \equiv 3(\bmod 4)$. This completes the proof of Proposition 3.2.

In above proof, we showed independently $H_{1} \not \equiv 0(\bmod q)$ and $H_{2} \not \equiv 0(\bmod q)$ under the assumption $q \equiv 3(\bmod 4)$. However, the second one can be deduced from the first if we apply more general results on Galois extensions and $p$-groups (see [16, Theorem 10.4 (a)]). Indeed, we have only to know and use the fact that $H_{1}$ is the relative class number of the subfield with degree 4 of $K_{p}$.

It is possible to discuss the $p$-divisibility of $H_{1}$ by means of Bernoulli numbers defined by the power series expansion

$$
\frac{x}{e^{x}-1}=\sum_{n=0}^{\infty} \frac{B_{n}}{n !} x^{n} \quad(|x|<2 \pi)
$$

As easily seen from the von Staudt-Clausen theorem, if $p$ is a prime with $p-1 \nmid n$, then $B_{n} \in \mathbb{Z}_{p}$, and if $p-1 \mid n$, then $p B_{n} \in \mathbb{Z}_{p}$, more precisely $p B_{n} \equiv-1(\bmod p)$. 
Proposition 3.3. Let $p=4 q+1$ be an odd prime with $q$ a prime. Then

$$
H_{1} \equiv \frac{1}{2} \cdot \frac{B_{(p+3) / 4}}{(p+3) / 4} \cdot \frac{B_{(3 p+1) / 4}}{(3 p+1) / 4} \quad(\bmod p) .
$$

Proof. Let $\omega$ be the Teichmüller character whose order is $p-1$. Since $\beta_{\omega^{n}} \equiv$ $\frac{1}{n+1} B_{n+1}(\bmod p)$ for a positive odd integer $n$ with $p-1 \nmid n+1$, we know that if $p=4 q+1$, then

$$
\begin{aligned}
H_{1} & =2 \mathcal{H}_{4}=2\left(\beta_{\omega^{q}} \beta_{\omega^{3 q}}\right)=2\left(-\frac{1}{2} B_{1, \omega^{q}}\right)\left(-\frac{1}{2} B_{1, \omega^{3 q}}\right) \\
& \equiv \frac{1}{2} \cdot \frac{B_{q+1}}{q+1} \cdot \frac{B_{3 q+1}}{3 q+1} \quad(\bmod p),
\end{aligned}
$$

which completes the proof of (3.5).

For above proof, we referred to some ideas written in the papers by Carlitz [2] and Metsänkylä [10]. As a matter of fact, there are many different proofs of (3.5) although the above one is very short and smart. It is of course possible to prove it by calculating $\mathcal{N}_{K_{4} / \mathbb{Q}}(f(i))$, where $f(x)=\sum_{k=0}^{p-2} g_{k} x^{k}$ as defined in Section 1 .

For this purpose, letting $s_{i}=\left(g g_{i}-g_{i+1}\right) / p \in \mathbb{Z}$, we consider the polynomial

$$
s(x)=s_{0}+s_{1} x+\cdots+s_{p-2} x^{p-2} .
$$

A basic relation between $f(x)$ and $s(x)$ is given by

$$
\frac{1}{p}(g x-1) f(x)=x s(x)+\frac{1}{p}\left(x^{p-1}-1\right) .
$$

As easily shown, if $m \geq 2$ is even and $p-1 \nmid m$, then we have

$$
\begin{aligned}
\frac{1}{p} f\left(g^{m-1}\right) & \equiv \frac{B_{m}}{m}+\frac{m-1}{g^{m}-1} q_{p}(g) \quad(\bmod p), \\
s\left(g^{m-1}\right) & \equiv \frac{g^{m}-1}{g^{m-1}} \cdot \frac{B_{m}}{m} \quad(\bmod p),
\end{aligned}
$$

where $q_{p}(g)=\left(g^{p-1}-1\right) / p$ is the Fermat quotient of $p$ with base $g$. Indeed, to deduce (3.7) we prepare the well-known congrunece (see, e.g., Agoh [1])

$$
\frac{B_{m}}{m} \equiv \frac{g^{m}}{g^{m}-1} q_{p}(g)-\sum_{i=1}^{p-1} g^{(m-1) i}\left[\frac{g^{i}}{p}\right] \quad(\bmod p),
$$

where $\left[g^{i} / p\right]$ is the greatest integer $\leq g^{i} / p$. By the logarithmic property of the Fermat quotient, we have $q_{p}\left(g^{n}\right) \equiv n q_{p}(g)(\bmod p)$ for any integer $n \geq 0$. Also since $\left[g^{i} / p\right]=\left(g^{i}-g_{i}\right) / p$ and $g_{p-1}=g_{0}=1$, it follows from (3.8) that

$$
\begin{aligned}
\frac{B_{m}}{m} & \equiv \frac{g^{m}}{g^{m}-1} q_{p}(g)-\frac{1}{p} \sum_{i=1}^{p-1} g^{m i}+\frac{1}{p} \sum_{i=1}^{p-1} g^{(m-1) i} g_{i} \\
& \equiv \frac{g^{m}}{g^{m}-1} q_{p}(g)-\frac{g^{m}}{g^{m}-1} q_{p}\left(p^{m}\right)+\frac{1}{p} f\left(g^{m-1}\right)+q_{p}\left(g^{m-1}\right) \\
& \equiv-\frac{m-1}{g^{m}-1} q_{p}(g)+\frac{1}{p} f\left(g^{m-1}\right) \quad(\bmod p),
\end{aligned}
$$


which shows the first congruence in (3.7). The second one can be shown from the first by taking $x=g^{m-1}$ in (3.6).

Now letting $\theta=\zeta_{p-1}$ for brevity, we get immediately from (3.6)

$$
\frac{1}{p}\left(g-\frac{1}{\theta}\right) f(\theta)=s(\theta) .
$$

If we set $\mathfrak{p}=(p, g-\theta)$ (the prime ideal of $\left.\mathcal{O}_{K_{p-1}}\right)$, then $g \equiv \theta(\bmod \mathfrak{p})$ and hence $g^{n} \equiv \theta^{n}(\bmod \mathfrak{p})$ for any $n \geq 0$. Taking this congruence into account, we obtain from (3.7) and (3.9) that, since $i=\theta^{(p-1) / 4}$ and $-i=\theta^{3(p-1) / 4}$,

$$
\begin{aligned}
H_{1} & =\frac{1}{2 p^{2}} \mathcal{N}_{K_{4} / \mathbb{Q}}(f(i))=\frac{1}{2 p^{2}}\left(\frac{p^{2}}{g^{2}+1} \mathcal{N}_{K_{4} / \mathbb{Q}}(s(i))\right) \\
& =\frac{1}{2\left(g^{2}+1\right)} s(i) s(-i)=\frac{1}{2\left(g^{2}+1\right)} s\left(\theta^{(p-1) / 4}\right) s\left(\theta^{3(p-1) / 4}\right) \\
& \equiv \frac{1}{2\left(g^{2}+1\right)} s\left(g^{(p-1) / 4}\right) s\left(g^{3(p-1) / 4}\right) \\
& \equiv M \cdot \frac{B_{(p+3) / 4}}{(p+3) / 4} \cdot \frac{B_{(3 p+1) / 4}}{(3 p+1) / 4}(\bmod p)
\end{aligned}
$$

where $M$ is given by, since $g^{(p-1) / 2} \equiv-1(\bmod p)$,

$$
M=\frac{\left(g^{(p+3) / 4}-1\right)\left(g^{(3 p+1) / 4}-1\right)}{2\left(g^{2}+1\right)} \equiv \frac{1}{2} \quad(\bmod p) .
$$

By the congruence (3.5), we can understand that $p \mid H_{1}$ if and only if at least one of pairs $(p,(p+3) / 4)$ and $(p,(3 p+1) / 4)$ is irregular. However it is unknown whether these pairs are irregular or not as well as the pair $(p,(p-1) / 2)$ related to the Ankeny-Artin-Chowla Conjecture on the fundamental unit of the real quadratic field $\mathbb{Q}(\sqrt{p})$ with $p \equiv 1(\bmod 4)$.

Concerning an upper bound for $H_{1}$, we can state

Proposition 3.4. Let $p=4 q+1$ be an odd prime with $q$ a prime. Then

$$
H_{1}<q^{2}=\left(\frac{p-1}{4}\right)^{2} \text {. }
$$

Proof. As stated in Section 2, $C=\left|2 U_{0} / p-q\right|$ and $D=\left|2 U_{1} / p-q\right|$, where $U_{a}=\sum_{k}^{(a)} k$ for $a=0,1$. Here we can calculate $U_{a}$ by means of a primitive root $g(\bmod p)$ as follows:

$$
\begin{aligned}
U_{a} & =\sum_{k}^{(a)} k=\sum_{j=0}^{q-1} g_{4 j+a}=\sum_{j=0}^{q-1}\left(g^{4 j+a}-\left[\frac{g^{4 j+a}}{p}\right] p\right) \\
& =\frac{g^{a}\left(g^{4 q}-1\right)}{g^{4}-1}-p \sum_{j=0}^{q-1}\left[\frac{g^{4 j+a}}{p}\right] .
\end{aligned}
$$

Using this we have

$$
\left|\frac{2 U_{a}}{p}-q\right|=2\left|\frac{g^{a}}{g^{4}-1} q_{p}(g)-\sum_{j=0}^{q-1}\left[\frac{g^{4 j+a}}{p}\right]-\frac{q}{2}\right| .
$$


Since $x-1<[x] \leq x$ for a real number $x$,

$$
\begin{aligned}
\sum_{j=0}^{q-1}\left(\frac{g^{4 j+a}}{p}-1\right) & =\frac{g^{a}}{g^{4}-1} q_{p}(g)-q<\sum_{j=0}^{q-1}\left[\frac{g^{4 j+a}}{p}\right] \\
& \leq \sum_{j=0}^{q-1} \frac{g^{4 j+a}}{p}=\frac{g^{a}}{g^{4}-1} q_{p}(g),
\end{aligned}
$$

and hence it follows that

$$
-\frac{q}{2}<\sum_{j=0}^{q-1}\left[\frac{g^{4 j+a}}{p}\right]-\frac{g^{a}}{g^{4}-1} q_{p}(g)+\frac{q}{2} \leq \frac{q}{2} .
$$

Consequently, from (3.10) we can show $\left|2 U_{a} / p-q\right|<q$ for each $a=0,1$ and this leads to $H_{1}=\left(C^{2}+D^{2}\right) / 2<q^{2}$ as indicated.

The size of $H_{2}$ is much larger than that of $H_{1}$ and it is surmised that $H_{2}$ grows more than exponentially with $p$. In fact, putting for a general prime $p$

$$
G(p)=2 p\left(\frac{p}{2 \pi^{2}}\right)^{(p-1) / 2},
$$

Kummer conjectured in 1851 that asymptotically $h_{p}^{-} \sim G(p)$ as $p \rightarrow \infty$, i.e. $\lim _{p \rightarrow \infty} h_{p}^{-} / G(p)=1$. The proof of this assertion is unknown, however Granville expanded heuristic arguments in [6] and proved that the Elliott-Halberstam and the Hardy-Littlewood Conjectures together imply that Kummer's Conjecture is false (see also Fung et al. [5]). On the other hand, Lepistö [9] proved the bounds

$$
\begin{gathered}
-\frac{1}{2} \log p-4 \log \log p-12.93-\frac{4.66}{\log p} \\
\leq \log \left(\frac{h_{p}^{-}}{G(p)}\right) \leq 5 \log \log p+15.49+\frac{4.66}{\log p},
\end{gathered}
$$

which shows that $h_{p}^{-}$grows rapidly. We cannot adopt the same argument as above because it is still open whether there exist infinitely many pairs $(p, q)$ of primes satisfying $p=4 q+1$, but we suppose from Proposition 3.4 that the growth of $H_{2}=h_{p}^{-} / H_{1}$ will be amazingly fast.

Here we want to enumerate concrete examples of $h_{p}^{-}, H_{1}, H_{2},(C, D)$ and $(S, T)$ for a few primes $p=4 q+1$ with $q$ a prime.

\section{Examples:}

- $(p, q)=(13,3): h_{p}^{-}=1$;

$H_{1}=1,(C, D)=(1,1) ; H_{2}=1,(S, T)=(5,2)$.

- $(p, q)=(29,7): h_{p}^{-}=2^{3}$; $H_{1}=1,(C, D)=(1,1) ; H_{2}=2^{3},(S, T)=(2 \cdot 11,2 \cdot 3)$.

- $(p, q)=(53,13): h_{p}^{-}=4889$; $H_{1}=1,(C, D)=(1,1) ; H_{2}=4889,(S, T)=\left(2^{5} \cdot 3 \cdot 5,47\right)$.

- $(p, q)=(149,37): h_{p}^{-}=3^{2} \cdot 149 \cdot 512966338320040805461$; $H_{1}=3^{2},(C, D)=(3,3) ; H_{2}=149 \cdot 512966338320040805461$, $(S, T)=(3 \cdot 149 \cdot 14489 \cdot 145091,2 \cdot 149 \cdot 1788084143)$. 
- $(p, q)=(173,43): h_{p}^{-}=5 \cdot 20297 \cdot 231169 \cdot 72571729362851870621 ;$

$H_{1}=5,(C, D)=(1,3)$;

$H_{2}=20297 \cdot 231169 \cdot 72571729362851870621$,

$(S, T)=\left(2 \cdot 3^{2} \cdot 2978771 \cdot 14703269237,131 \cdot 16477 \cdot 55695394459\right)$.

It seems from numerical tables (e.g., [8]) and other inspections that the following statements hold, although we do not have any definite ideas how to prove them.

(1) $H_{1}<p$ and hence $p \nmid H_{1}$.

(2) $p\left|h_{p}^{-} \Longleftrightarrow p\right| H_{2} \Longleftrightarrow p \mid S$ and $p \mid T$.

(3) $2 \nmid h_{p}^{-} \Longrightarrow l^{2} \nmid H_{2}$ for any odd primes $l$ (i.e. $H_{2}$ is square-free).

It is possible to give an upper bound for $\log H_{1} / \log p$ as a deduction from the Brauer-Siegel Theorem (cf. [16, Chapter 4]). We cannot say exactly now, but such an estimation may be useful to confirm (1).

In this paper, we discussed some arithmetic properties of factors of $h_{p}^{-}$only for the case when $p$ has the form $p=4 q+1$ with $q$ a prime. It is possible to extend above results to more general cases for primes $p=2^{n} q+1$ where $n \geq 3$ and $q$ is an odd prime.

Acknowledgments. The author would like to express many thanks to Tauno Metsänkylä for his valuable comments and to Tetsuya Taniguchi for his generous help concerning numerical verifications by computer.

\section{REFERENCES}

[1] T. Agoh, Congruences involving Bernoulli numbers and Fermat-Euler quotients, J. Number Theory 94 (2002), 1-9.

[2] L. Carlitz, The first factor of the class number of a cyclic field, Canad. J. Math. 6 (1954), 23-26.

[3] D. Davis, Computing the number of totally positive circular units which are squares, J. Number Theory 10 (1978), 1-9.

[4] D. R. Estes, On the parity of class number of the field of qth root of unity, Rocky Mountain J. Math. 19 (1989), 675-682.

[5] G. Fung, A. Granville and H. C. Williams, Computation of the first factor of the class number of cyclotomic fields, J. Number Theory 42 (1992), 297-312.

[6] A. Granville, On the size of the first factor of the class number of a cyclotomic field, Invent. Math. 100 (1990), 321-338.

[7] D. H. Lehmer, Prime factors of cyclotomic class numbers, Math. Comp. 31 (1977), 599-607.

[8] D. H. Lehmer and J. M. Masley, Table of cyclotomic class number $h^{*}(p)$ and their factors for $200<p<521$, Math. Comp. 32 (1978), 577-582.

[9] T. Lepistö, On the growth of the first factor of the class number of the prime cyclotomic field, Ann. Acad. Sci. Fenn. Ser. AI 577 (1974), 1-21.

[10] T. Metsänkylä, A congruence for the class number of a cyclic field, Ann. Acad. Sci. Fenn. Ser. AI 472 (1970), 1-11.

[11] T. Metsänkylä, On prime factors of the relative class numbers of cyclotomic fields, Ann. Univ. Turku. Ser. AI 149 (1971), 1-8.

[12] T. Metsänkylä, Some divisibility results for the cyclotomic class number, Number Theory (Liptovský Ján, 1995), Tatra Mountains Math. Publ. 11 (1997), 59-68.

[13] T. Metsänkylä, On the parity of the class number of real abelian fields, Proc. of the 13th Czech and Slovak International Conference on Number Theory (Ostravice, 1997), Acta Math. Inf. Univ. Ostraviensis 6 (1996), 159-166. 
[14] P. Ribenboim, Classical Theory of Algebraic Numbers, Springer, Berlin-Heidelberg-New York, 2001.

[15] P. Stevenhagen, Class number parity for the pth cyclotomic field, Math. Comp. 63 (1994), 773-784.

[16] L. C. Washington, Introduction to Cyclotomic Fields, Springer, Berlin-Heidelberg-New York, 1982.

Takashi Agoh, Department of Mathematics, Tokyo University of Science, 2641 Yamazaki, Noda, Chiba 278-8510, Japan

e-mail: agoh_takashi@ma.noda.tus.ac.jp 